\title{
Intraperitoneal corticosteroids for recurrent ascites in patients with Fontan circulation: Initial clinical experience
}

\author{
Fred $\mathrm{Wu}^{1}$, Anne Valente ${ }^{1}$, Peter Nigrovic ${ }^{2}$, Anna Rutherford ${ }^{2}$, and Michael Singh ${ }^{1}$ \\ ${ }^{1}$ Boston Children's Hospital \\ ${ }^{2}$ Brigham and Women's Hospital
}

June 25, 2020

\begin{abstract}
Individuals with single-ventricle congenital heart disease who are palliated to a Fontan circulation are at risk for heart failure and liver disease, with recurrent ascites being one potentially debilitating cause of late morbidity. Although ascites associated with heart failure or liver failure is usually characterized by a high serum-ascites albumin gradient (SAAG), we have observed multiple instances of ascites in Fontan patients with low SAAG, suggesting an inflammatory process. We present three cases in which recalcitrant ascites severely and adversely impacted quality of life, and describe our initial experience with intraperitoneal corticosteroids in this setting.
\end{abstract}

\section{Background}

In the Fontan procedure, a palliative operation for single-ventricle congenital heart disease, the systemic veins are connected directly to the pulmonary circulation without a subpulmonary ventricle. Individuals with Fontan circulation are at risk for heart failure and liver disease, with recurrent ascites being one cause of late morbidity. ${ }^{1}$ Ascites associated with heart failure or liver failure is usually characterized by a serumascites albumin gradient (SAAG)[?]1.1. ${ }^{2}$ However, we have observed multiple instances of ascites in Fontan patients with low SAAG, suggesting a possible inflammatory process. We present three such cases in which recalcitrant ascites severely and adversely impacted quality of life, and describe our initial experience with intraperitoneal corticosteroids in this setting.

\section{Presentation and Clinical Data}

Patient 1

Patient 1 was a 30-year-old woman with double-outlet right ventricle and mitral atresia who underwent atriopulmonary Fontan palliation at 4 years old. At age 28, she underwent Fontan conversion for recurrent arrhythmia. Afterward, she developed ascites requiring frequent paracentesis.

Her evaluation included (1) blood tests showing normal liver synthetic function; (2) liver biopsy showing "established cirrhosis," and (3) cardiac catheterization showing cardiac index (CI) of $4.5 \mathrm{~L} / \mathrm{min} / \mathrm{m}^{2}$, Fontan pressure of $14 \mathrm{mmHg}$, pulmonary capillary wedge pressure (PCWP) of $9 \mathrm{mmHg}$, pulmonary vascular resistance (PVR) of 1.3 Wood units (iWU), and a hepatic vein pressure gradient (HVPG) of $4 \mathrm{mmHg}$. At the first paracentesis, the fluid was non-chylous; SAAG was 0.8 , and fluid protein was $5.3 \mathrm{~g} / \mathrm{dL}$. Fluid Gram stain, cultures, and cytology were negative for infection or malignancy. Serum C-reactive protein (CRP) was $15.8 \mathrm{mg} / \mathrm{L}$.

Given her poor prognosis, she agreed to a trial of intraperitoneal steroids when she started requiring paracentesis more than once a month despite escalating doses of diuretic. After therapeutic paracentesis during which $5.2 \mathrm{~L}$ of fluid was removed, $500 \mathrm{mg}$ of triamcinolone hexacetonide was introduced into the peritoneal 
space. The procedure was well tolerated. She did not require paracentesis again until 45 later; $6 \mathrm{~L}$ of fluid was removed, after which another $500 \mathrm{mg}$ of triamcinolone hexacetonide was administered intraperitoneally. Again, there were no immediate complications. One week later, she experienced sudden death at home. Her family declined autopsy.

\section{Patient 2}

Patient 2 was a 34-year-old woman with mitral atresia and pulmonary atresia who underwent atriopulmonary Fontan palliation at age 4. At 17 years old, she underwent conversion to a lateral tunnel Fontan due to recurrent atrial arrhythmias that persisted despite radiofrequency ablation.

She first developed ascites at age 22. At paracentesis, the SAAG was 0.7 , and fluid protein was $6.2 \mathrm{~g} / \mathrm{dL}$. She had a markedly elevated serum CRP of $169.6 \mathrm{mg} / \mathrm{L}$. Evaluation by rheumatology was inconclusive, but given her significant illness, she was treated with prednisone and methotrexate. After 2 years without ascites, the prednisone was weaned off. Methotrexate was stopped another 3 years later, but her ascites recurred shortly afterward.

Evaluation for causes of ascites included (1) blood tests showing normal liver synthetic function; (2) abdominal CT showing a cirrhotic-appearing liver without discrete lesions; (3) esophagogastroduodenoscopy showing no varices; (4) liver biopsy showing hepatic fibrosis and regenerative hepatocellular changes; (5) peritoneal biopsy showing mesothelium-lined soft tissue without significant pathologic change; and (6) cardiac catheterization showing CI of $1.9 \mathrm{~L} / \mathrm{min} / \mathrm{m}^{2}$, Fontan pressure of $8 \mathrm{mmHg}$, PCWP of $4 \mathrm{mmHg}$, PVR of $1.3 \mathrm{iWU}$, and a HVPG of $3 \mathrm{mmHg}$. Within 1 year, she was requiring paracentesis every $2-3$ weeks, each time with a low SAAG. Fluid triglycerides were $50 \mathrm{mg} / \mathrm{dL}$, and Gram stain, cultures, and cytology were negative. Her CRP was $173.0 \mathrm{mg} / \mathrm{L}$. Based on these findings and on the temporal association of ascites with discontinuation of methotrexate, it was felt that an anti-inflammatory agent would be appropriate. Given concerns about toxicity related to long-term use of these agents, we decided to try intraperitoneal steroids.

After removal of $5 \mathrm{~L}$ of fluid by paracentesis, $500 \mathrm{mg}$ of triamcinolone hexacetonide was introduced into the peritoneal space. She experienced post-procedural hyperglycemia that persisted for one week; the procedure was otherwise well tolerated. After 3 weeks, she was readmitted for ascites and underwent a second paracentesis with removal of $4.3 \mathrm{~L}$ of fluid followed again by administration of $500 \mathrm{mg}$ of triamcinolone hexacetonide into the peritoneal space. Again, she experienced post-procedural hyperglycemia that resolved after a few days.

She did not require paracentesis again until 20 weeks after the second administration of intraperitoneal triamcinolone. By 8-1/2 months, she began presenting frequently for paracentesis. After the sixth paracentesis in a 3-month period, she again received $500 \mathrm{mg}$ of intraperitoneal triamcinolone hexacetonide after removal of $4.5 \mathrm{~L}$ of ascites. Although she did not experience hyperglycemia this time, adrenocorticotropic hormone stimulation testing 2 weeks later showed evidence of adrenal insufficiency. She was started on low-dose hydrocortisone. She required paracentesis two more times over the next 6 weeks but then did not need paracentesis again until 45 weeks later. During this time, her hydrocortisone was weaned without any adverse effects. Although her ascites became intractable, no more intraperitoneal triamcinolone was given due to the previous adverse effects; she underwent paracentesis 43 more times over the next 3 years, including 23 times in her final year. She ultimately developed Candida peritonitis and died within 2 weeks of diagnosis.

\section{Patient 3}

Patient 3 was a woman with double-inlet left ventricle and pulmonary atresia who underwent atriopulmonary Fontan palliation at age 23. At 47, she underwent conversion to an extracardiac conduit Fontan due to recurrent atrial arrhythmias. She remained stable until presenting at age 56 with new-onset ascites.

Evaluation for causes of ascites included (1) blood tests showing normal liver synthetic function; (2) liver biopsy showing bridging fibrosis and focal nodule formation consistent with cirrhosis, and (3) cardiac catheterization showing CI of $2.7 \mathrm{~L} / \mathrm{min} / \mathrm{m}^{2}$, Fontan pressure of $16 \mathrm{mmHg}$, PCWP of $12 \mathrm{mmHg}$, PVR of $1.7 \mathrm{iWU}$, and HVPG of $1 \mathrm{mmHg}$. At her initial paracentesis, the fluid was non-chylous. SAAG was 
0.7 , and fluid protein was $5.1 \mathrm{~g} / \mathrm{dL}$. Fluid Gram stain, cultures, and cytology were negative for infection or malignancy. Serum CRP was $16.8 \mathrm{mg} / \mathrm{L}$.

Nine months after paracentesis, she was involved in a motor vehicle accident that resulted in hemoperitoneum. She was hospitalized for 5 days during which she underwent paracentesis on two occasions and was transfused a total of 3 units of red blood cells and 4 units of fresh frozen plasma. Although her hemoperitoneum resolved, she developed recurrent ascites. She agreed to a trial of intraperitoneal triamcinolone. After $3.4 \mathrm{~L}$ of serous fluid was removed by paracentesis, $150 \mathrm{mg}$ of triamcinolone hexacetonide was introduced into the peritoneal space. The procedure was well tolerated. She underwent paracentesis again 3-1/2 months later but then never again accumulated significant ascites. She died 15 months after this last paracentesis from complications following surgery for epicardial pacemaker lead placement.

\section{Comment}

Patients with a Fontan circulation are at risk for both liver disease and congestive heart failure. Ascites is one complication of this that is seen in roughly one-third of adults post-Fontan and can itself adversely affect Fontan hemodynamics. ${ }^{1,3}$ Recurrent ascites poses a management challenge due to limited efficacy of diuretic therapy. In some patients, repeated paracentesis may become necessary but comes with a risk for complications including protein depletion, hypovolemia, electrolyte abnormalities, infection, and bleeding.

Ascites related to heart failure or liver disease is typically expected to have a SAAG[?]1.1. In cases where SAAG is low, an alternative or coexisting process should be considered. Chylous ascites has been described in congenital heart disease and can be associated with low SAAG, but can be recognized by high levels of fluid triglyceride and white blood cells with a lymphocytic predominance. Several studies have also suggested that inflammation plays a role in Fontan morbidity. ${ }^{4,5}$

Having excluded other etiologies for ascites in our patients and given the poor prognosis associated with intractable ascites, we felt it appropriate to consider a novel intervention targeting inflammation. Intraperitoneal triamcinolone has been described for ascites related to lupus, hemodialysis, and malignancy. ${ }^{6-11} \mathrm{We}$ speculated that intraperitoneal administration of a large-molecule steroid would allow a higher dose of steroid to the vessels responsible for the ascites while limiting systemic absorption.

In each case, triamcinolone hexacetonide was administered following large-volume paracentesis. Sometimes a second dose was given after the subsequent paracentesis. Patients 1 and 3 tolerated triamcinolone administration without clinical evidence of systemic steroid absorption, and the rate of ascites accumulation appeared to decrease (Table 1). While Patient 1 died shortly afterward from presumed arrhythmic arrest, Patient 3 experienced complete resolution of ascites until her death from unrelated causes over a year later. Patient 2 also experienced a decrease in the rate of ascites accumulation after intraperitoneal triamcinolone, but due to adverse effects, it was not felt to be an option when her ascites recurred. She eventually died from complications of frequent paracentesis.

Given the lack of efficacy of diuretics in this clinical situation and the risks associated with frequent paracentesis, we feel that further studies exploring the efficacy and toxicity of intraperitoneal steroids in Fontan patients with low-SAAG ascites using objective metrics would be of great interest to physicians caring for this unique population.

\section{Compliance with Ethical Standards}

Funding: No research funds were used for this study.

Conflict of Interest: The authors declare that they have no conflicts of interest.

Ethical approval: All procedures performed in studies involving human participants were in accordance with the ethical standards of the institutional and/or national research committee and with the 1964 Helsinki declaration and its later amendments or comparable ethical standards.

Informed consent: Informed consent was obtained from all individual participants included in the study. 


\section{Acknowledgements}

The authors would like to acknowledge the clinical and administrative staff of the Boston Adult Congenital Heart (BACH) and Pulmonary Hypertension Program for their support in the preparation of this manuscript.

TABLE 1: Paracentesis frequency and volume pre- and post-triamcinolone.

\begin{tabular}{|c|c|c|c|c|c|}
\hline & $\begin{array}{l}\text { Triamcinolone } \\
\text { dose given }\end{array}$ & $\begin{array}{l}\text { Paracentesis } \\
\text { frequency } 90 \mathrm{~d} \\
\text { pre- } \\
\text { triamcinolone }\end{array}$ & $\begin{array}{l}\text { Paracentesis } \\
\text { frequency 90d } \\
\text { post- } \\
\text { triamcinolone }\end{array}$ & $\begin{array}{l}\text { Ascites volume } \\
\text { removed 90d } \\
\text { pre- } \\
\text { triamcinolone } \\
\text { (L) }\end{array}$ & $\begin{array}{l}\text { Ascites volume } \\
\text { removed 90d } \\
\text { post- } \\
\text { triamcinolone } \\
(\mathrm{L})\end{array}$ \\
\hline Patient 1 & $500 \mathrm{mg} \times 2$ & 4 & $1^{*}$ & 28.7 & $6.0^{*}$ \\
\hline $\begin{array}{l}\text { Patient } 2 \text { First } \\
\text { administration }\end{array}$ & $500 \mathrm{mg} \times 2$ & 6 & 1 & 19.3 & 4.3 \\
\hline $\begin{array}{l}\text { Patient } 2 \text { Second } \\
\text { administration }\end{array}$ & $500 \mathrm{mg} \times 1$ & 5 & 3 & 21.3 & 9.1 \\
\hline Patient 3 & $150 \mathrm{mg} x 1$ & 4 & 1 & 11.3 & 2.2 \\
\hline
\end{tabular}

*Patient died 52 days after triamcinolone

\section{REFERENCES:}

1. Wu FM, Kogon B, Earing MG, et al. Liver health in adults with Fontan circulation: A multicenter cross-sectional study. J Thorac Cardiovasc Surg.2017;153(3):656-664.

2. Pare P, Talbot J, Hoefs JC. Serum-ascites albumin concentration gradient: a physiologic approach to the differential diagnosis of ascites. Gastroenterology.1983;85(2):240-244.

3. Rajpal S, Opotowsky AR, Rutherford A, Wu F. Hemodynamic Effects of Paracentesis in a Patient With a Fontan Circulation. World journal for pediatric \&3 congenital heart surgery. 2019;10(3):357-359.

4. Mainwaring RD, Lamberti JJ, Hugli TE. Complement activation and cytokine generation after modified Fontan procedure. Ann Thorac Surg. 1998;65(6):1715-1720.

5. Ostrow AM, Freeze H, Rychik J. Protein-losing enteropathy after fontan operation: investigations into possible pathophysiologic mechanisms. Ann Thorac Surg.2006;82(2):695-700.

6. Bansal VK, Popli S, Ing TS, Hano JE, Geis WP, Kamadana MR. Intraperitoneal infusion of a nonabsorbable steroid in hemodialysis-associated ascites. J Dial.1978;2(4):379-385.

7. Diaz-Buxo JA, Chandler JT, Farmer CD, Walker PJ. Intraperitoneal infusion of non-absorbable steroids in the treatment of ascites and sterile peritonitis. J Dial.1980;4(1):43-50.

8. Jenkin RP, Bamford R, Patel V, Kelly L, Stern S. The use of intraperitoneal triamcinolone acetonide for the management of recurrent malignant ascites in a patient with non-Hodgkin's lymphoma. J Pain Symptom Manage. 2008;36(5):e4-5.

9. Mackey JR, Wood L, Nabholtz J, Jensen J, Venner P. A phase II trial of triamcinolone hexacetanide for symptomatic recurrent malignant ascites. J Pain Symptom Manage.2000;19(3):193-199.

10. Shoji T, Takatori E, Miura Y, et al. Pilot study of intraperitoneal administration of triamcinolone acetonide for cancerous ascites in patients with end-stage gynecological cancer. Int $J$ Gynecol Cancer. 2014;24(6):1093-1097.

11. Zhou QG, Yang XB, Hou FF, Zhang X. Successful treatment of massive ascites with intraperitoneal administration of a steroid in a case of systemic lupus erythematosus.Lupus. 2009;18(8):740-742. 